Document downloaded from:

http://hdl.handle.net/10251/105490

This paper must be cited as:

Martinez-Ferri, JE.; Juste Vidal, BJ.; Ortiz Moragón, J.; Martorell Alsina, SS.; Verdú Martín, GJ. (2017). Air radon equilibrium factor measurement in a Waste Water Pre-Treatment Plant. Radiation Physics and Chemistry. 140:20-24. doi:10.1016/j.radphyschem.2017.03.011

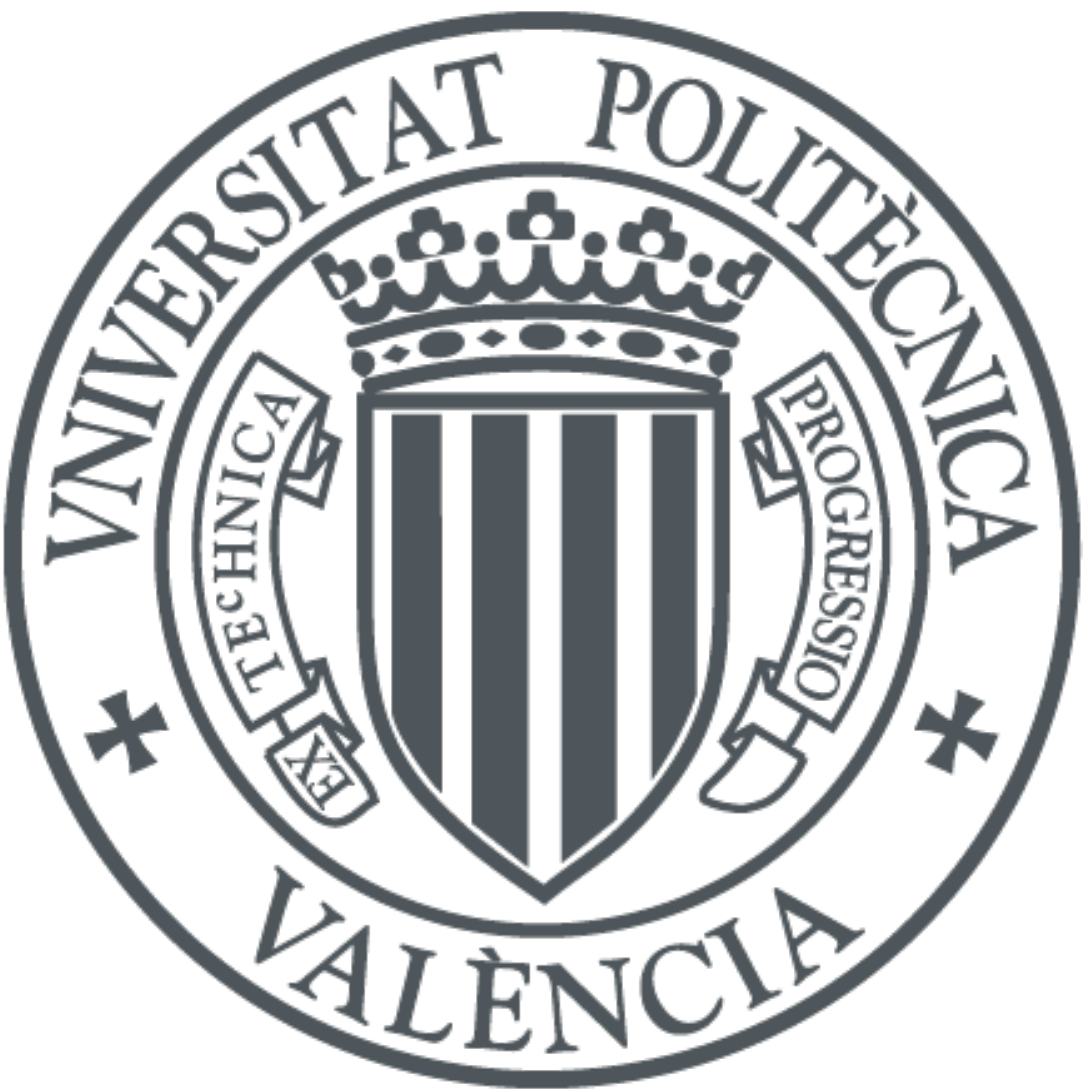

The final publication is available at

http://doi.org/10.1016/j.radphyschem.2017.03.011

Copyright Elsevier

Additional Information 


\title{
Air Radon equilibrium factor measurement in a Waste Water Pre-Treatment Plant
}

\author{
J. E. Martinez ${ }^{\mathrm{a}}$, B. Juste ${ }^{\mathrm{a}}$, J. Ortiz ${ }^{\mathrm{b}}$, S. Martorell ${ }^{\mathrm{b}}$ G. Verdu ${ }^{\mathrm{a}}$ \\ ${ }^{a}$ Instituto de Seguridad Industrial, Radiofísica y Medioambiental (ISIRYM). \\ Universitat Politècnica de València, Camí de Vera s/n. 46022, Spain \\ bLaboratorio de Radioactividad Ambiental. Universitat Politècnica de València, Camí de Vera \\ s/n. 46022, Spain
}

Keywords: Radon, equilibrium factor, gamma spectroscopy, Bateman equation, radon progeny.

\begin{abstract}
We analyze in this paper a Waste Water Pre-Treatment Plant (WWTP) located at the Mediterranean coast with air radon concentration above Spanish action level (600 Becquerel per cubic meter).

This paper presents a method for radon equilibrium determination by gamma spectrometry measuring of the radon progeny concentrations in the air, in order to estimate WWTP workers effective dose more exactly.

The method is based on simultaneous sampling of air through a filter paper and alpha spectrometry measurement of radon activity concentration in the air.

According to the measured radon activity concentration in the air of $368 \pm 45 \mathrm{~Bq} / \mathrm{m}^{3}$ the equilibrium factor between radon and progenies is estimated to be $F=0.27$, which is in good agreement with expected values.
\end{abstract}

\section{Introduction}

Equilibrium factor between radon and short-lived progenies is of special importance for dose assessment from radon inhalation and it should be determined in each radon monitoring.

Direct measurements of radon decay products concentration are difficult to perform and rather limited. Therefore, they are estimated taking into account the equilibrium between radon and its decay products.

According to UNSCEAR (UNSCEAR., 2000), radon Equilibrium-Equivalent Concentration (EEC) is calculated:

$$
E E C_{R n-222}=0.105 C_{A P o-218}+0.515 C_{A P b-214}+0.38 C_{A B i-214}
$$


Where $C_{A P o-218}, C_{A P b-214}$ and $C_{A B i-214}$ are the activity concentrations of the short-lived decay products in air.

The equilibrium factor is calculated then as the ratio of the EEC to the radon activity concentration $\left(C_{A \mathrm{~m}}\right)$ :

$$
F=\frac{E E C_{R n-222}}{C_{A m}}
$$

According to the conversion coefficients of ICRP65: "Protection Against Radon-222 at Home and at work" (ICRP., 1993), effective dose per unit exposure at work (mSv per $\mathrm{mJhm}^{-3}$ ) is 1.4 and conversion factor of radon progeny exposition is $2.22 \cdot 10^{-6} \mathrm{mJhm}^{-3}$ per $\mathrm{Bqhm}^{-3}$ (assuming equilibrium factor (F) 0.4). ICRP 65 suggests a rounded value for the equilibrium factor of 0.4 for indoor environments. This estimated value may vary in different situations, since it is influenced by environmental factors such as pressure, temperature, humidity, etc. To obtain a precise dose calculation caused by radon and its progeny, it is necessary to know the value of the equilibrium factor in each case.

Different techniques have been developed for the measurement of the equilibrium factor. In literature, most used methods are based on the detection of gross alpha activities that bring a great deal of uncertainty and error (Leung et al., 2006; Mingli, Y et al., 2010), or “ $\beta$ count” (Singh, K et al., 2006).

One particular place where indoor radon concentrations can exceed national guidelines (Nuclear Safety Council., 2011) is in Waste Water Pre-Treatment Plants (WWTPs), where treatment processes may contribute to ambient airborne concentrations. When the water containing radium is aerated or backwashed, elevated concentrations of radon are released. Therefore, WWTP operators may be exposed to an increased risk of Rn-222 inhalation, due to the movement of gas from the water to air while water treatment processes are being carried out (Juste, B., 2015).

The equilibrium factor is measured in a WWTP plant located at the Mediterranean coast. In this plant, the two workers spend an average of 2.5 hours a day inside the treatment plant according to the permanency registries given. The workers are exposed to an annual average of radon concentration that occasionally exceeds the proposed nowadays limitation level of $600 \mathrm{~Bq} / \mathrm{m}^{3}$.

In this paper, we propose a novelty mathematical resolution (for avoid the absence of the Po-218, due to its short period) method for $F$ calculation. It consists in alpha spectrometry measurement of the activity concentration of radon in the air. Simultaneously, the radon progeny activity concentrations are determined by gamma spectrometry of the filter paper. To do that, we used a pump air suction through a filter where the progeny of radon were trapped (Forkapić, S., 2011). The advantage of the gamma spectroscopy method is its accuracy and fast determination of radionuclide activities. 


\section{Measurement equipment}

Air sampler F\&J DF-14ME (Figure 1) with fiber glass filter paper of high collection efficiency of $\varepsilon=98 \%$ was used for aerosol sampling. The pump flow velocity was adjusted to a value of $v=1 \mathrm{e}^{-3} \mathrm{~m}^{3} / \mathrm{s}(60 \mathrm{~L} / \mathrm{min})$. During the experiment radon short lived daughters attached to aerosols were collected on the fixed filter paper.

Even if the suction flow with this pump is not high, as the suction time is 22 minutes (because of radon daughters period decay), and radon concentrations are over $300 \mathrm{~Bq} / \mathrm{m}^{3}$, measurable quantities of Po-218, Pb-214 and Bi-214 are adhered to the filter.

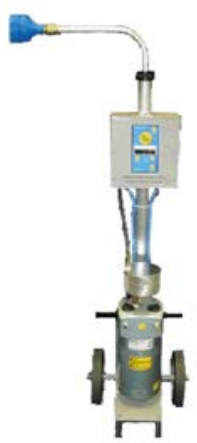

Figure 1. Air Sampler with glass fiber filter paper.

On the other hand, radon activity concentration was measured during 3 days by alpha spectrometer RAD-7 (Durridge Company, USA) (Figure 2) (Durridge., 2015), which enables continuous and direct reading of radon concentration in air. RAD7 Detector is based on electrostatic collection of alpha-emitters with spectral analysis using a passive Ion-implanted Planar Silicon detector.

The measurement procedure with this meter has been verified by means of the participation in several national intercomparison (Gutierrez, J., 2016).

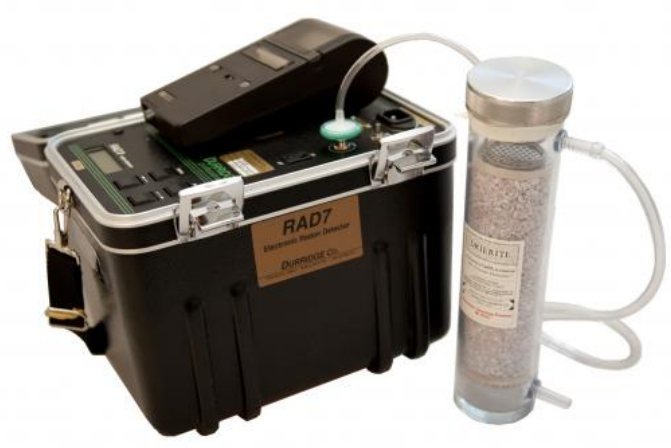

Figure 2. Alpha spectrometer RAD-7 (Durridge Company). 
After the suction, the filter paper which is put in a sealed container to avoid the contamination, was sent to the Environmental Laboratory,(LRA, Laboratorio de Radioactividad Ambiental) is an accredited laboratory in gamma spectrometry of the Polytechnic University of Valencia, and measured by gamma spectrometry in four successive measurements of 1000 seconds duration. These sequential measures performed allowed to see the radon progeny peaks evolution. 1000 seconds measurement time was selected to obtain quality spectra, and time is short enough to perform three other sequential measures.

The gamma spectrometry measurements were performed by an Ortec GMX 40 Germanium detector, with an extended range from $6 \mathrm{keV}$ to $3 \mathrm{MeV}$ in original lead shield with a wall thickness of $10 \mathrm{~cm}$ and $2 \mathrm{keV}$ resolution (1.33 MeV Co-60).

\section{Methods and methodology}

We describe here the algorithm method for radon progenies determination in the air at the start of suction.

The air suction time $t_{s}$ is 1320 seconds. Once the fiber filter is collected, it is transported ( $t_{t} 7200$ seconds) to the LRA where the radon descendants are measured in the germanium detector. In this laboratory four sequential measures ( $t_{m} 1000$ seconds) are performed.

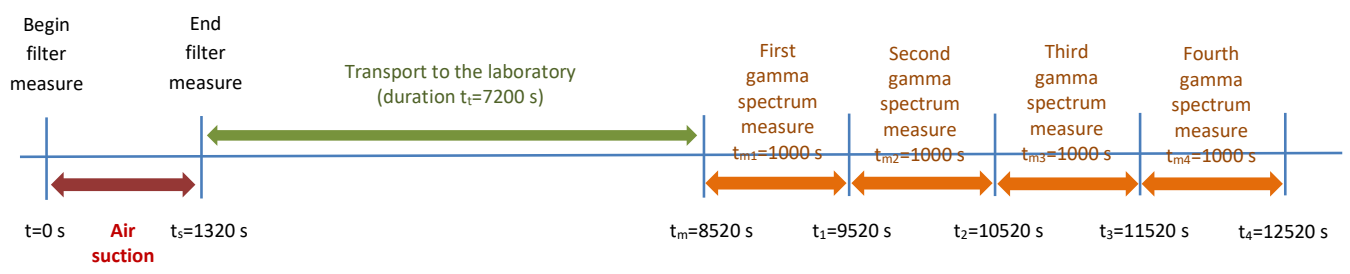

Figure 3. Temporal evolution of filter exposure and measures.

In order to connect the results of gamma spectrometry measurements of the filter paper and the radon progeny concentrations in the air at the start of suction, it is necessary to take into account decay corrections during the time of suction $t_{s}$, cooling time $t_{t}$ after the suction but before the measurements and during the measurements $t_{m}$, because these periods of time are not negligible in comparison to the short life times of radon daughters (Po-218 (3 min), Pb-214 (26.8 min) and Bi-214 (19.9 min)).

After the time $t_{s}$ elapsed from the beginning to stopping of suction, the number of radon progeny atoms Po-218, Pb-214 and Bi-214 collected on the filter paper $N_{P o}, N_{P b}$ and $N_{B i}$ changed according to differential equations (eqs. 3-5), assuming that at the beginning of suction there were no radon progenies on the filter paper $N_{P o}(0)=0, N_{P b}(0)=0$ and $N_{B i}(0)=0$ :

$$
\frac{d N_{P o}}{d t_{R}}=C_{P o} v \varepsilon-\lambda_{P o} N_{P o}
$$




$$
\begin{aligned}
& \frac{d N_{P b}}{d t_{R}}=C_{P b} v \varepsilon+\lambda_{P o} N_{P o}-\lambda_{P b} N_{P b} \\
& \frac{d N_{B i}}{d t_{R}}=C_{B i} v \varepsilon+\lambda_{P b} N_{P b}-\lambda_{B i} N_{B i}
\end{aligned}
$$

$C_{P o}, C_{P b}$ and $C_{B i}$ are the radon progeny concentrations in the air at the start of suction. These values correspond to the unknown quantity to be obtained from this equation system, where $v=0.001 \mathrm{~m}^{3} / \mathrm{s}$ is the pump suction flow and $\varepsilon=0.98$ is the filter efficiency.

$\lambda_{i}$ are the corresponding decay constant parameters $\lambda_{P o}=0.003787 \mathrm{~s}^{-1}, \lambda_{P b}=0.000431 \mathrm{~s}^{-1}$, $\lambda_{B i}=0.0005805 \mathrm{~s}^{-1}$.

To solve the Bateman equations system, a Matlab routine module has been developed. The resolution of this equations system provides a set of new equations dependent on the progeny radon concentrations.

$$
\begin{gathered}
N_{P o}^{\prime}(0)=0.25703 C_{P o} \text { (6) } \\
N_{P b}^{\prime}(0)=0.823161 C_{P o}+0.986511 C_{P b} \quad(7) \\
N_{B i}^{\prime}(0)=0.171644 C_{P o}+0.23904 C_{P b}+0.903873 C_{B i}
\end{gathered}
$$

Where $N^{\prime}{ }_{P o}(0)=N_{P o}\left(t_{s}\right), N{ }_{P b}(0)=N_{P b}\left(t_{s}\right), N^{\prime}{ }_{B i}(0)=N_{B i}\left(t_{s}\right)$.

After the suction of the air through the filter paper, the radon progeny atoms captured on the filter continue decaying during the cooling and measuring time, and the number of atoms change in accordance with other set of differential equations (9-11). The initial conditions for this system of equations were obtained by solving the first system of differential equations (6-8):

$$
\begin{aligned}
& \frac{d N_{P o}^{\prime}}{d t}=-\lambda_{P o} N_{P o}^{\prime} \\
& \frac{d N_{P b}^{\prime}}{d t}=\lambda_{P o} N_{P o}^{\prime}-\lambda_{P b} N_{P b}^{\prime} \\
& \frac{d N_{B i}^{\prime}}{d t}=\lambda_{P b} N_{P b}^{\prime}-\lambda_{B i} N_{B i}^{\prime}
\end{aligned}
$$

The number of decayed nuclei of $\mathrm{Pb}-214$ and Bi-214 during the measurement can be connected with the gamma spectrometry detected results of filter paper measured:

$$
N_{r}=\frac{N_{d}}{\epsilon_{d} p_{\gamma}}
$$


Where, $N_{d}$ is the area under the photopeak, $N_{r}$ is the number of decayed nuclei during the time of measurement $t_{m}, \varepsilon_{d}$ is photopeak detection efficiency and $p_{\gamma}$ is the $\gamma$-ray emission probability.

Solving equations (9) and (10) we obtain:

$$
N_{P b}^{\prime}(t)=N_{P b}^{\prime}(0) e^{-\lambda_{P b} t}-\frac{\lambda_{P o}}{\lambda_{P b}-\lambda_{P o}} N_{P o}^{\prime}(0)\left[e^{-\lambda_{P b} t}-e^{-\lambda_{P o} t}\right]
$$

Detected decays are actually the difference between the not decayed nuclei after the decay time $t=t_{t}$ and the not decayed nuclei after the measurement time $t=t_{t}+t_{m}$.

$$
N_{r}=N^{\prime}\left(t=t_{t}\right)-N^{\prime}\left(t=t_{t}+t_{m}\right)
$$

Then the detected decays for the $\mathrm{Pb}-214$ are given by

$$
N_{r}(P b-214)=e^{-\lambda_{P b} t_{t}}\left[1-e^{-\lambda_{P b} t_{m}}\right]\left[N_{P b}^{\prime}(0)-\frac{\lambda_{P o}}{\lambda_{P b}-\lambda_{P O}} N_{P O}^{\prime}(0)\right]+\frac{\lambda_{P o}}{\lambda_{P b}-\lambda_{P o}} N_{P O}^{\prime}(0) e^{-\lambda_{P o} t_{t}}\left[1-e^{-\lambda_{P o} t_{m}}\right]
$$

To obtain the values $N^{\prime}{ }_{\mathrm{Pb}}(0)$ and $N^{\prime}{ }_{\mathrm{Po}}(0)$ we need at least two measures at $t_{t}=t_{1}$ and $t_{t}=t_{1}+t_{m}$. The main disadvantage of this method is that the first measurement time should be after few minutes of the final time of suction. For example, if $t_{1}$ is about 15 minutes after $\mathrm{t}_{\mathrm{s}}$, it is not possible to discriminate $N^{\prime}{ }_{\mathrm{Pb}}(0)$ and $N^{\prime}{ }_{\mathrm{Po}}(0)$ because $e^{-\lambda_{P o} t_{t}} \simeq 0$. Nevertheless, in our case, the high transport time of the filter to the laboratory makes it not possible to register this difference.

The solution of equation (11) is given by

$$
N_{B i}^{\prime}=N_{B i}^{\prime}(0) e^{-\lambda_{B i}}-\frac{\lambda_{P b} N_{P b}^{\prime}(0)}{\lambda_{B i}-\lambda_{P b}}\left[e^{-\lambda_{B i} t}-e^{-\lambda_{P b t}}\right]-\frac{\lambda_{P o} \lambda_{P b}}{\lambda_{P b}-\lambda_{P o}} N_{P o}^{\prime}(0)\left[e^{-\lambda_{P b} t}-e^{-\lambda_{B i} t}\right]
$$

In this case,

$$
\begin{gathered}
N_{r}(B i-214)=\left[N_{B i}^{\prime}(0)-\frac{\lambda_{P b} N_{P b}^{\prime}(0)}{\lambda_{B i}-\lambda_{P b}}+\frac{\lambda_{P o} \lambda_{P b}}{\lambda_{P b}-\lambda_{P o}} N_{P o}^{\prime}(0)\right] e^{-\lambda_{B i} t_{t}}\left[1-e^{-\lambda_{B i} t_{m}}\right]+ \\
{\left[\frac{\lambda_{P b} N_{P b}^{\prime}(0)}{\lambda_{B i}-\lambda_{P b}}-\frac{\lambda_{P o} \lambda_{P b} N_{P o}^{\prime}(0)}{\lambda_{P b}-\lambda_{P o}}\right] e^{-\lambda_{P b} t_{t}}\left[1-e^{-\lambda_{P b} t_{m}}\right]}
\end{gathered}
$$

For the equation (17), if $N^{\prime}{ }_{\mathrm{Po}}(0)$ and $N^{\prime}{ }_{\mathrm{Pb}}(0)$ are known, it is possible to obtain $N^{\prime}{ }_{B i}(0)$, but, as we have said before $N^{\prime}{ }_{P o}(0)$ and $N{ }^{\prime}{ }_{P b}(0)$ are linked by equation (15) , thus an approximation is made. Therefore $C_{P o}$ is approximated as $F \cdot C_{A}(R n-222) / \lambda_{P o}$ being $F$ an initial equilibrium factor estimated and $C_{A}(R n-222)$ the activity concentration of radon measure with the alfa spectrometer RAD7. Then, $N^{\prime}{ }_{P o}(0)=C_{P o} / 0.25704$. In this way $N{ }^{\prime}{ }_{P b}(0)$ can be obtained from equation (15), and $N{ }^{\prime}{ }_{B i}(0)$ is also obtained from equation (17). Afterwards, from equations (7) and (8) we obtained the concentrations $C_{P b}$ and $C_{B i}$. The next step is to find the activity concentration of Po-218, Pb-214 and Bi-214. 


$$
\begin{aligned}
& C_{A}(P o-218)=\lambda_{P o} C_{P o} \\
& C_{A}(P b-214)=\lambda_{P b} C_{P b} \\
& C_{A}(B i-214)=\lambda_{B i} C_{B i}
\end{aligned}
$$

Finally, the equilibrium factor is given by equations (1) and (2).

Once the calculations are done, a new value of equilibrium factor for Po-218 is updated, and the equation (15) and (17) are applied again to obtain a new value of $N^{\prime}{ }_{B i}(0)$ and $N^{\prime} P b(0)$ and subsequently a new equilibrium factor is attained. The iterative process converges in a few steps. It must be mentioned that only one gamma spectrometry measure is needed, the other measures are used to correct errors.

\section{Results}

Due to the long time of the transport to LRA, we have developed a novelty mathematical resolution method (explained above) for avoid the absence of Po-218 when we did the measurements.

In order to check our methodology, the algorithm was first applied to measurements obtained by Forkapić, S (Forkapić, S., 2011). The equilibrium factor obtained with our methodology was $F=0.55$, which was in good agreement with the equilibrium factor obtained by S. Forkapic in his work $F=0.56$.

In our case the filter was placed in a Petri little plastic box for gamma spectrometry measurements with HPGe. Measures were successive repeated four times (1000 seconds duration each of them). The next table (Table 1) displays the obtained results. The net areas under the photopeaks of $351.9 \mathrm{keV}$ (Pb-214) and $609.3 \mathrm{keV}$ (Bi-214) obtained experimentally are listed. The measurement of Po-218 are null.

Table 1: Experimental results for four successive measurements of filter paper (Net area value).

\begin{tabular}{|c|c|c|l|l|}
\hline & & & $\begin{array}{l}\mathrm{Pb}-214 \\
\mathrm{~N}_{\mathrm{d}}(351.9 \mathrm{keV})\end{array}$ & $\begin{array}{l}\mathrm{Bi}-214 \\
\mathrm{~N}_{\mathrm{d}}(609.3 \mathrm{keV}) \\
\mathrm{t}_{\mathrm{t}}(\mathrm{s})\end{array}$ \\
\hline 7200 & 1000 & 8200 & 224 & 303 \\
\hline 8200 & 1000 & 9200 & 149 & 226 \\
\hline 9200 & 1000 & 10200 & 97 & 164 \\
\hline 10200 & 1000 & 112000 & 65 & 115 \\
\hline
\end{tabular}

As it can be seen, Po-218 is not present in the above table because the Polonium half-life is shorter than the time needed to attempt the first measuremt of the sample. Therefore, Polonium did not remain in the sample by the time of measurement. 
Figure 5 shows the obtained spectrum measured for the first time. It displays $\mathrm{Pb}-214$ (351.9 KeV) and Bi-214 (609.3 KeV) peaks. Figure 6 shows the third measured 3000 seconds after the first one.

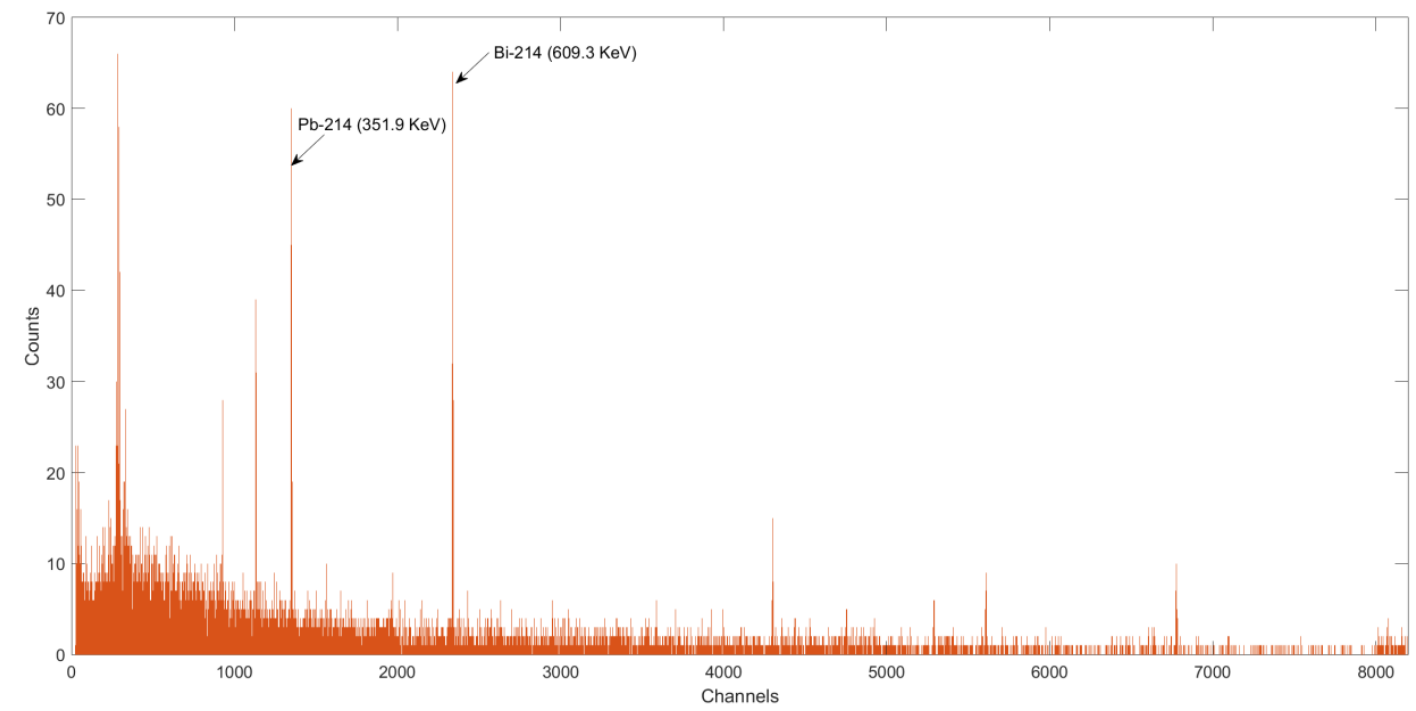

Figure 4. First gamma spectrum measure (Gammavision).

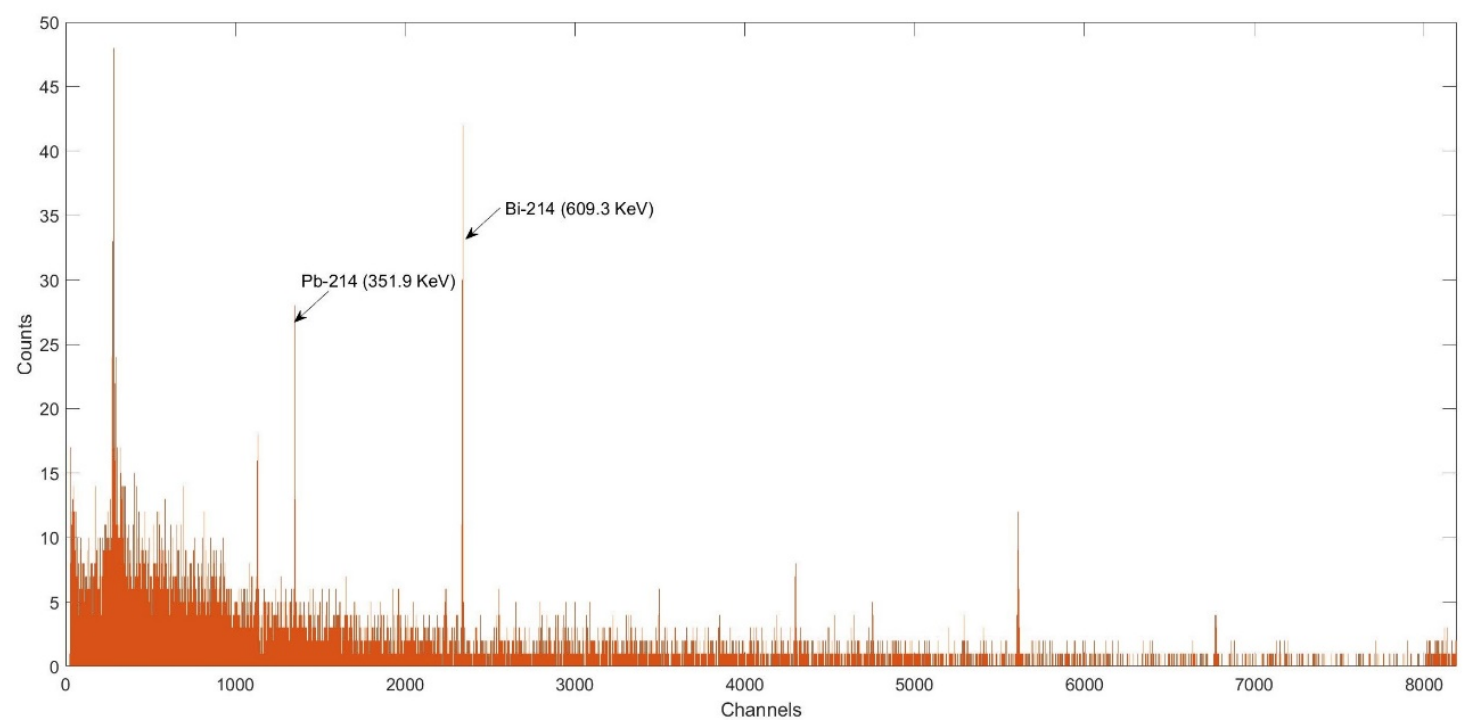

Figure 5. Gamma spectrum measure at $\mathrm{t}=10200 \mathrm{~s}$ (Gammavision).

Figure 7 represents the evolution of gross peak areas mentioned before (Figure 5 and 6) of the four filter gamma spectrum measures, of the filter. 


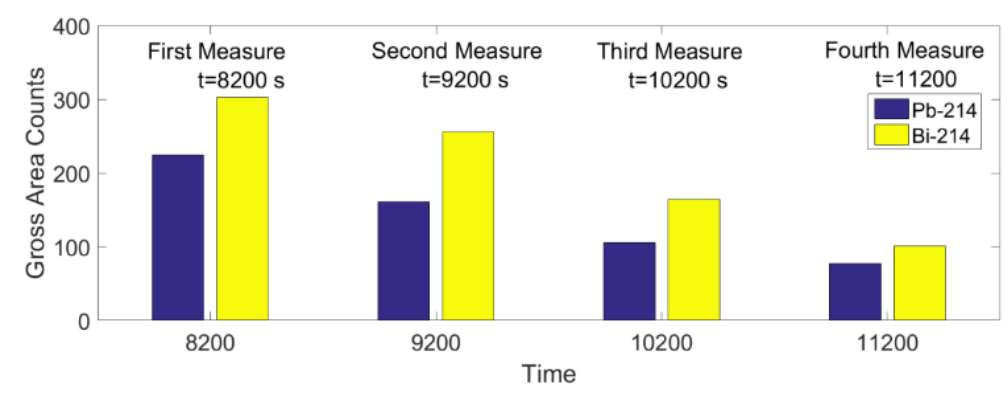

Figure 6. Evolution of peak areas.

Applying the solving algorithm developed, the equations system is solved. Therefore the activity concentrations of radon progeny in the air at the start of sampling were calculated:

$$
\begin{gathered}
C_{A}(P o-218)=\lambda_{P o} \cdot C_{P o}=104.2 \mathrm{~Bq} / \mathrm{m}^{3} \\
C_{A}(P b-214)=\lambda_{P b} \cdot C_{P b}=161.4 \mathrm{~Bq} / \mathrm{m}^{3} \\
C_{A}(B i-214)=\lambda_{B i} \cdot C_{B i}=32.6 \mathrm{~Bq} / \mathrm{m}^{3}
\end{gathered}
$$

Finally, according to measured radon activity concentration in the air of $C_{R n-222}=368 \pm 6$ $\mathrm{Bq} / \mathrm{m}^{3}$, the equilibrium factor between radon and progenies was estimated to $F=0.27$.

\section{Conclusions}

Generally, a default value of 0.4 is used for the equilibrium factor in living areas according to ICRP 65 (ICRP., 1993) and UNSCEAR (UNSCEAR., 2000). While this is usually a reasonable assumption, there are cases where the equilibrium factor can significantly differ from 0.4 due to various housing and environmental factors.

Because the effective dose depends strongly on the $F$ value, it is important for risk assessment to know the normal range of this factor at specific settings. We describe in this paper a methodology to measure equilibrium factor in a Waste Water Pre-treatment plant, where high concentrations of radon affect workers.

Based on the developed methodology, an equilibrium factor of 0.27 is obtained. This result indicates that the $F$ recommended by ICRP (ICRP., 1993) and UNSCEAR (UNSCEAR., 2000) overestimates the doses received by workers of this Waste Water Pre-Treatment Plant. The lower value of the equilibrium factor obtained is due to the air extraction system, which renews the air 10 times per hour.

In future works, we will analyze the dependence of the F-factor on seasonal variations.

\section{References}

Durridge Radon Instrumentation., 2015. RAD 7 Radon Detector, user manual. DURRIDGE Company.

Forkapić, S., Mrđa, D., Vesković, M., Todorović, N., Bikit, K., Nikolov, J., Hansman, J., 2012 . Radon equilibrium measurement in the air. In Paper presented at the First East European Radon Symposium-FERAS. 
Gutierrez, J, L., Sainz, S., 2016. Intercomparacion de monitores de gas radón en condiciones de campo. Saelices el Chico (España). Editorial de la Universidad de Cantabria.

ICRP., 1993. Protection Against Radon-222 at Home and at Work. ICRP Publication 65. Ann. ICRP 23 (2).

Juste, B., Ortiz, J., Verdú, G., Martorell, S., 2015. Air radon concentration decrease in a waste water treatment plant. Radiat Prot Dosimetry 2015; 164 (4): 497-501. http://dx.doi.org/10.1093/rpd/ncv329.

Leung S,Y,Y., Nikezic, D., Yu,K,N., 2006. Passive monitoring of the equilibrium factor inside a radon exposure chamber using bare LR 115 SSNTDs. Nuclear Inst. and Methods in Physics Research A 564 319-323. http://dx.doi.org/10.1016/j.nima.2006.04.031.

Mingli, Y. et al., 2010. Proceedings of the 3rd Symposium of Natural Radiation Exposure and Control. pp 601-606, (Chinese).

Nuclear Safety Council (Consejo de Seguridad Nuclear, CSN)., 2011. Instruction IS-33,on the radiological criteria for the protection against exposure to natural radiation. CSN.

Singh, K., Singh, S., Mehra, R., Singh, M., Sahota, H, S., Papp, Z., 2006. Measurement of radon and thoron progeny outdoors in Malout, India, using grab aerosol sampling and beta counting. Radiation Measurements,vol.41,no,1,pp.108-111. http://dx.doi.org/10.1016/j.radmeas.2005.03.002.

UNSCEAR., 2000. Sources and effects of ionizing radiation, New York: UNITED NATIONS, ANNEX B. 